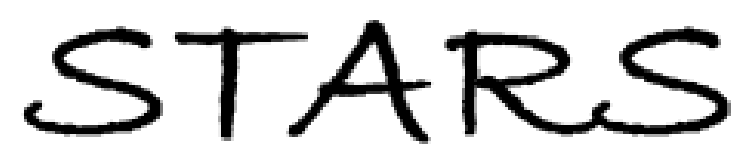

University of Central Florida

STARS

$1-1-2013$

\title{
Blueshifted continuum peaks from filamentation in the anomalous dispersion regime
}

\author{
Magali Durand \\ University of Central Florida \\ Khan Lim \\ University of Central Florida \\ Vytautas Jukna \\ Erik McKee \\ University of Central Florida \\ Matthieu Baudelet \\ University of Central Florida
}

Find similar works at: https://stars.library.ucf.edu/facultybib2010

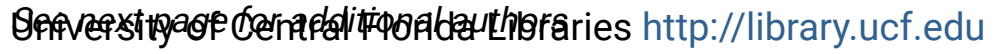

This Article is brought to you for free and open access by the Faculty Bibliography at STARS. It has been accepted for inclusion in Faculty Bibliography 2010 s by an authorized administrator of STARS. For more information, please contactSTARS@ucf.edu.

\section{Recommended Citation}

Durand, Magali; Lim, Khan; Jukna, Vytautas; McKee, Erik; Baudelet, Matthieu; Houard, Aurèlien; Richardson, Martin; Mysyrowicz, Andrè; and Couairon, Arnaud, "Blueshifted continuum peaks from filamentation in the anomalous dispersion regime" (2013). Faculty Bibliography 2010s. 3922. https://stars.library.ucf.edu/facultybib2010/3922

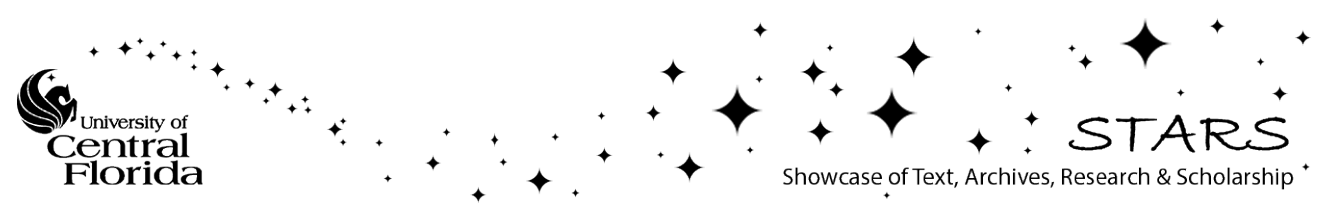




\section{Authors}

Magali Durand, Khan Lim, Vytautas Jukna, Erik McKee, Matthieu Baudelet, Aurèlien Houard, Martin Richardson, Andrè Mysyrowicz, and Arnaud Couairon 


\title{
Blueshifted continuum peaks from filamentation in the anomalous dispersion regime
}

\author{
Magali Durand, ${ }^{1,2, *}$ Khan Lim, ${ }^{1}$ Vytautas Jukna ${ }^{3}$ Erik McKee, ${ }^{1}$ Matthieu Baudelet, ${ }^{1}$ Aurélien Houard,${ }^{2}$ Martin Richardson, ${ }^{1}$ \\ André Mysyrowicz, ${ }^{2}$ and Arnaud Couairon ${ }^{3}$ \\ ${ }^{1}$ Townes Laser Institute, CREOL-The College of Optics and Photonics, University of Central Florida, Orlando, Florida 32816, USA \\ ${ }^{2}$ Laboratoire d'Optique Appliquée, ENSTA ParisTech, Ecole Polytechnique, CNRS, 91761 Palaiseau, France \\ ${ }^{3}$ Centre de Physique Théorique, CNRS, Ecole Polytechnique, F-91128 Palaiseau, France
}

(Received 14 February 2013; published 15 April 2013)

\begin{abstract}
Filamentation in the anomalous dispersion regime is experimentally shown to give rise to an extreme blueshifted continuum peak in the visible region even when the filament is formed by near-infrared pulses. Measurements and numerical simulations allow us to identify this peak as an axial component of the conical emission. Its features are characterized and quantitatively reproduced by the effective three-wave mixing model.
\end{abstract}

DOI: 10.1103/PhysRevA.87.043820

PACS number(s): 42.65.Jx, 42.65.Re

The propagation of high-peak-power laser pulses (above the megawatt level for solid materials) through transparent media gives rise to a nonlinear propagation regime called filamentation [1,2]. This regime is characterized by an intense and narrow beam surrounded by a low-intensity reservoir. The intense core interacts with matter over distances exceeding the typical diffraction length. The use of filaments has been proposed for a wide range of applications such as terahertz generation [3], discharge guiding [4], atmospheric analysis [5], and few-cycle pulse generation [6]. Pulses with peak power exceeding the megawatt level at near-infrared wavelengths (1-2.5 $\mu \mathrm{m})$ can be easily generated by the use of an optical parametric amplifier (OPA), and have allowed experimental investigations on filamentation in the anomalous dispersion regime since 2004 [7-12]. Moll and Gaeta have shown that anomalous group velocity dispersion induces a transfer of energy into the collapse region and a substantial increase of the characteristic distances at which multiple collapses occur [7]. Porras et al. [8,9] have interpreted measurements of filamentation and conical emission in the normal and anomalous dispersion regimes as a manifestation of the nonsolitary stationary solutions to the nonlinear Schrödinger equation. These solutions, called nonlinear $\mathrm{X}$ or $\mathrm{O}$ waves, arise from the self-focusing dynamics in Kerr media with nonlinear losses and normal or anomalous dispersion, respectively. Saliminia et al. [10] and Faccio et al. [11] have shown the emergence of a broad peak in the visible part of the supercontinuum spectra generated by a $1.5-\mu \mathrm{m}$ filament. Recent reports on supercontinuum emission from filaments at longer wavelengths confirm this observation while showing that filamentation in the anomalous dispersion regime gives rise to a broader continuum of over 3.3 octaves that could ideally self-compress to a single-cycle pulse [13-16].

In this article, we present measurements of supercontinuum emission by filamentation in the anomalous dispersion region of fused silica. By varying the initial wavelength for filamentation in the near-infrared region, we uncover the physical mechanism at the origin of the conical emission in the visible region.

From the first set of experiments, spectra obtained from filamentation in the anomalous dispersion regime of fused

\footnotetext{
*Corresponding author: magali.durand@creol.ucf.edu
}

silica are shown in Fig. 1. The propagation of 35-fs, $1.9-\mu \mathrm{m}$ laser pulses generates a broad continuum around the fundamental wavelength, as well as an intense component in the visible close to the third-harmonic wavelength. Similar peaks close to the third harmonic were previously reported in measurements of supercontinuum from filaments in a YAG crystal [13]. They were not interpreted as the third harmonic but as the axial component of the angularly resolved $(\theta, \lambda)$ spectrum that exhibits a fish-shaped tail formed by scattering of the incident pulse from the induced refractive index change $[11,17]$. In Ref. [14], the pump wavelength was changed from 1.2 to $2.1 \mu \mathrm{m}$ but the central wavelength of the visible peak was not observed to change significantly, ruling out third-harmonic generation as a key process responsible for the formation of the visible peak. The authors interpreted the generation of the "anti-Stokes component" in terms of an interference model [14-16].

Our measurements reported in this article show a significant blueshift of the central wavelength of the visible peak when the laser wavelength was redshifted. We interpret the results in terms of the effective three-wave mixing model (ETWM) [18].

In the second set of experiments, the initial filamentation wavelength was varied from 1.2 to $2.4 \mu \mathrm{m}$. The laser source was an OPA (Opera F, Coherent) pumped by a titaniumsapphire chirped-pulse amplified laser system that delivered up to $1.1-\mathrm{mJ}, 100-\mathrm{fs}$ pulses at $800 \mathrm{~nm}$ with a repetition rate of $1 \mathrm{kHz}$ (Spitfire, Spectra-Physics). The pulses at the output of the OPA had energies up to $100 \mu \mathrm{J}$, at $100 \mathrm{fs}$. The laser beam was focused with a $50-\mathrm{cm}$ focal length lens inside a 3-cm-long fused silica hexagonal rod (Hexagonal Light Pipe, Edmund). The measurements taken by three spectrometers were combined to cover the spectral range from 298 to $2571 \mathrm{~nm}$ [HR 4000 (298-756 nm), HR 4000 (711-889 nm) and NIRQUEST (853-2571 nm), from Ocean Optics]. The spectral response of the spectrometers was calibrated using a NIST-calibrated tungsten lamp. The on-axis pulse spectrum was measured via an optical fiber. The energy at each laser wavelength was chosen experimentally to be as close to the critical power of filamentation as possible by visually determining the threshold for supercontinuum generation; this energy was around $3.5 \mu \mathrm{J}$ for central wavelengths from 1.2 to $1.6 \mu \mathrm{m}$, then $5 \mu \mathrm{J}$ for $1.7 \mu \mathrm{m}, 7 \mu \mathrm{J}$ for $1.8 \mu \mathrm{m}, 7.2 \mu \mathrm{J}$ for $1.9 \mu \mathrm{m}, 7.1 \mu \mathrm{J}$ for $2 \mu \mathrm{m}, 7.3 \mu \mathrm{J}$ for $2.1 \mu \mathrm{m}, 9.5 \mu \mathrm{J}$ for $2.2 \mu \mathrm{m}, 18 \mu \mathrm{J}$ for $2.3 \mu \mathrm{m}$, and $12.5 \mu \mathrm{J}$ for $2.4 \mu \mathrm{m}$. The 


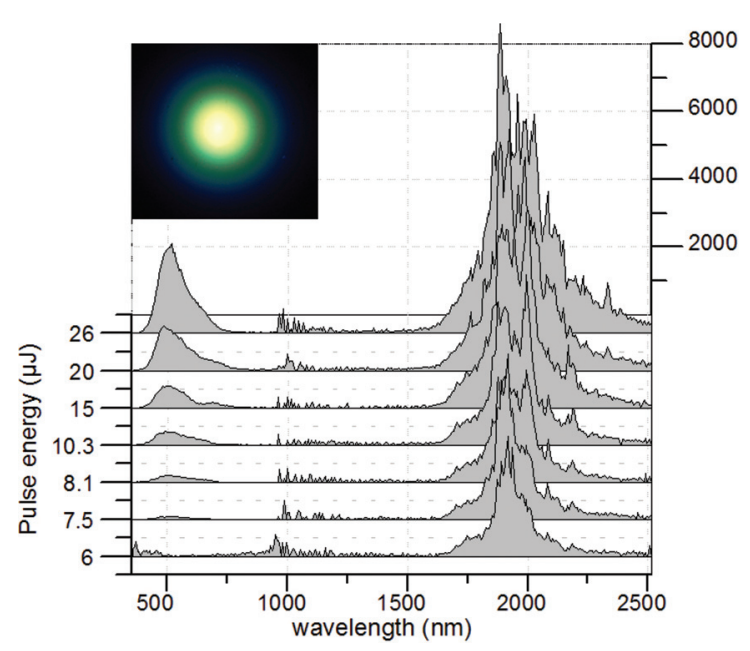

FIG. 1. (Color online) Supercontinuum measured at the center of the conical emission after filamentation in $3 \mathrm{~cm}$ of fused silica of a 1.9- $\mu \mathrm{m}, 35$-fs pulse at different input energies. Inset: Photo of the conical emission at $15 \mu \mathrm{J}$.

resulting spectra are shown in Fig. 2. The visible part of the conical emission is clearly shifted to shorter wavelengths when the initial filamenting pulse is varied to longer wavelengths, ruling out third-harmonic generation as the mechanism at the origin of the visible emission.

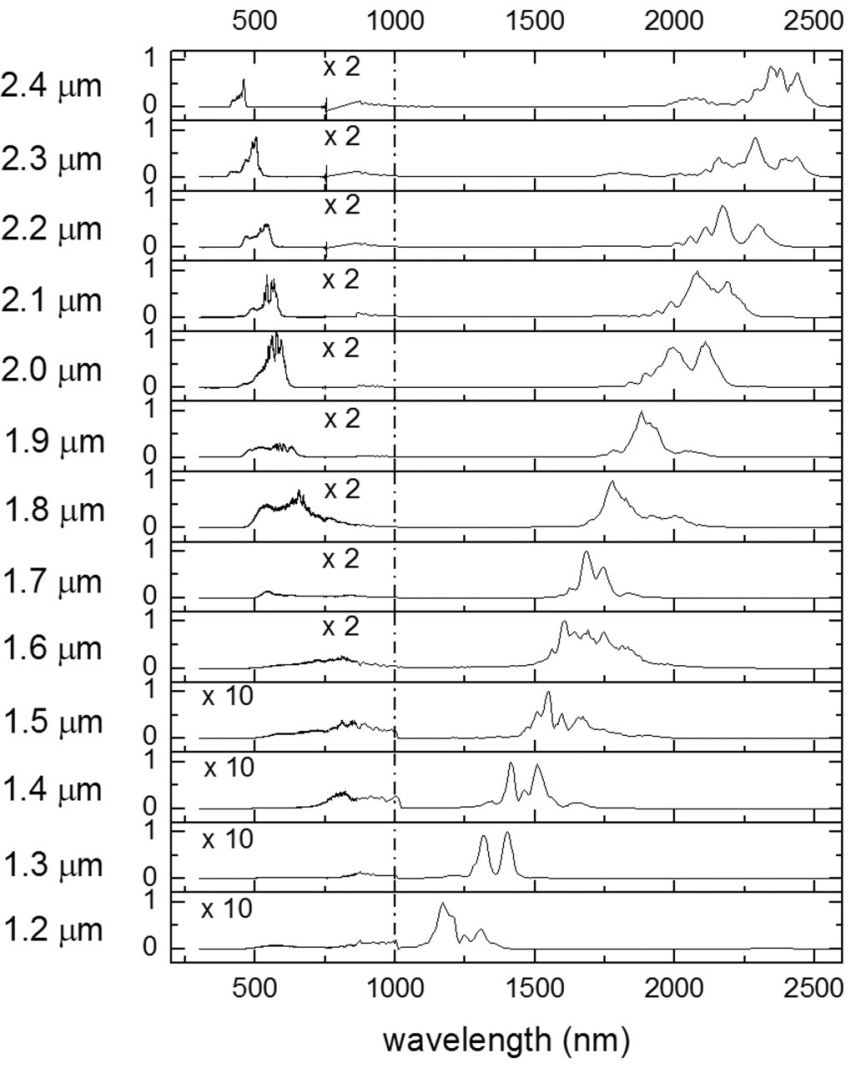

FIG. 2. Spectra measured at the center of the conical emission after filamentation in a 4-cm-thick fused silica sample. The central wavelength used for filamentation ranged from 1.2 to $2.4 \mu \mathrm{m}$. The visible part of the spectra up to $1 \mu \mathrm{m}$ is magnified by a factor of 10 for $\lambda_{0}(1.2-1.5 \mu \mathrm{m})$ and by a factor of 2 for $\lambda_{0}(1.6-2.4 \mu \mathrm{m})$.
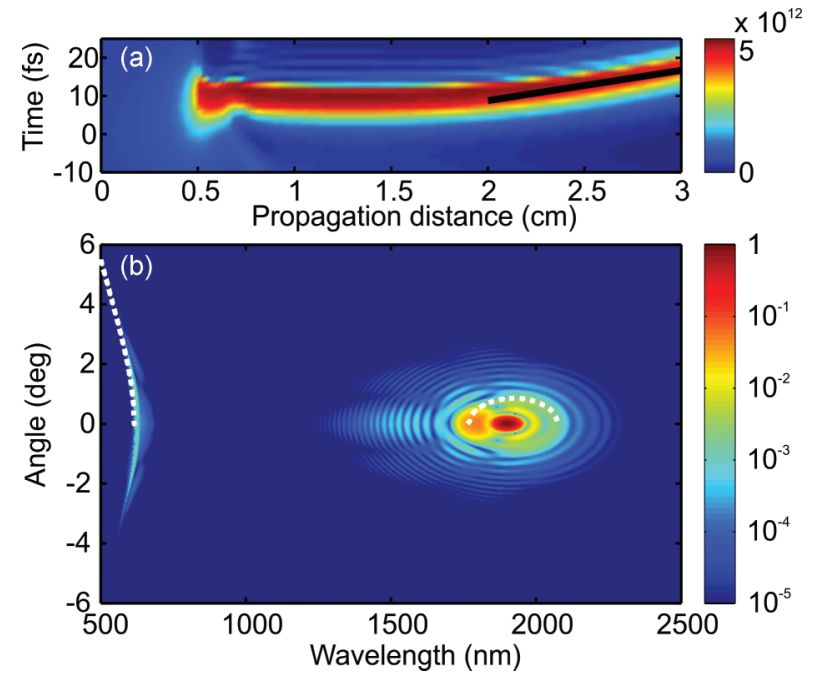

FIG. 3. (Color online) (a) Computed pulse temporal evolution during filamentation in a 3 -cm-long fused silica sample. The input pulse has central wavelength $\lambda_{0}=1.9 \mu \mathrm{m}$, duration $70 \mathrm{fs}$, and energy $7.2 \mu \mathrm{J}$. The black line marks the slope for calculating the velocity of the intensity peak. (b) $(\theta, \lambda)$ spectrum obtained by numerical simulation. The dashed curves show the fit with Eq. (2).

In order to interpret the results using the ETWM model, we first note that the nonlinear polarization is generated by welllocalized intensity peaks of the pulse. In the case of anomalous dispersion, numerical simulations show that, in a range of initial powers above the critical power for self-focusing, the pulse undergoes filamentation but does not split and therefore retains the profile of a single peak with a propagation velocity $v_{p}$ that can be larger or smaller than the group velocity $v_{g}$. Figure 3 shows the result of a numerical simulation with input parameters corresponding to the experimental results shown in Fig. 2, i.e., for a 70-fs, 1.9- $\mu \mathrm{m}, 7.2-\mu \mathrm{J}$ pulse. (See Refs. [12,19,20] for details of the numerical scheme and fused silica material properties.) The velocity $v_{p}$ can be determined by calculating the local slope $d t / d z$ and from the relation $v_{p}=\left(1 / v_{g}+d t / d z\right)^{-1}$. A subluminal velocity $\left(v_{p}<v_{g}\right)$ is obtained for positive $d t / d z$. The solid black line superimposed in Fig. 3(a) indicates a quasiconstant $d t / d z$ over the last centimeter of propagation, corresponding to a velocity $\alpha=1 / v_{p}=1.0002 k_{0}^{\prime}$. The traveling perturbation of the refractive index acts as a polarization wave with a frequency $\omega-\omega_{0}$ that scatters the incident pulse of frequency $\omega_{0}$ into a scattered wave of frequency $\omega$ and transverse wave number $k_{\perp}$. This process is associated with the phase-matching condition [18]:

$$
\Delta k \equiv k_{0}+\frac{\left(\omega-\omega_{0}\right)}{v_{p}}-k_{z}\left(\omega, k_{\perp}\right) \approx 0 .
$$

The longitudinal wave number of the scattered wave fulfills the condition $k_{\perp}^{2}+k_{z}^{2}=k^{2}(\omega)$, where $k(\omega)$ denotes the dispersion relation in fused silica [20]. Introduction of the dispersion relation in the effective three-wave mixing relation (1) allows us to find the loci of the supported new frequencies:

$$
k_{\perp}= \pm \sqrt{k^{2}(\omega)-\left(k_{0}-\Delta k+\alpha \Omega\right)^{2}},
$$

where $\Omega \equiv \omega-\omega_{0}$ and $\alpha=1 / v_{p}$. 


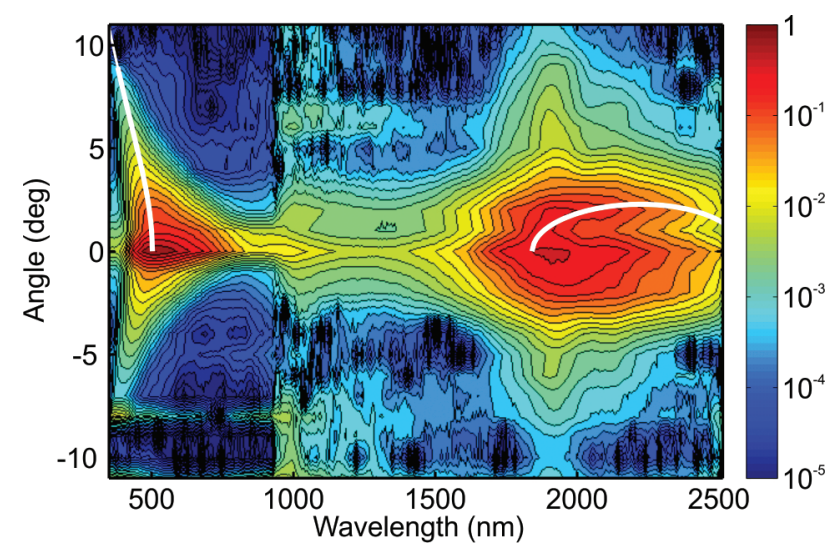

FIG. 4. (Color online) Angular distribution of the conical emission, after filamentation of $1.9-\mu \mathrm{m}, 40-\mathrm{fs}, 26-\mu \mathrm{J}$ pulses in $3 \mathrm{~cm}$ of fused silica. This figure has a logarithmic scale. The white curves show the fit with Eq. (2).

From the simulation shown in Fig. 3(a), the far-field pattern of the pulse was reconstructed. Figure 3(b) presents the angular repartition $(\theta, \lambda)$ of the spectrum. It consists of elliptical structures around the pump wavelength (the so-called $\mathrm{O}$ waves) and a tail in the visible region that represents the conical emission part of the supercontinuum, corresponding to the blueshifted peak measured in the experiments. These features match perfectly with the angular distribution of wavelengths given by Eq. (2), depicted by the dashed white curves with the velocity parameter $\alpha=1.0002 k_{0}^{\prime}$ as determined from Fig. 3(a) and $\Delta k=0.25 \mathrm{~mm}^{-1}$. The conical emission in the visible region is reproducible independently of the value of the phase mismatch $\Delta k$, whereas each ring of the Oshaped structure around the pump is populated for a specific value of $\Delta k$, reflecting the interaction length over which the pulse propagates with quasiconstant peak intensity and velocity.

Other than by analyzing the results of numerical simulation, the velocity of the nonlinear polarization can also be determined experimentally by fitting the angularly resolved spectrum with Eq. (2). Measurements of $(\theta, \lambda)$ spectra were performed only in our first set of experiments. Figure 4 presents the $(\theta, \lambda)$ spectrum measured for a 35-fs pump pulse at $\lambda_{0}=1.9 \mu \mathrm{m}$. The solid white curves represents a fit obtained with Eq. (2) and the values $\alpha=1.0035 k_{0}^{\prime}$ and $\Delta k=0.5 \mathrm{~mm}^{-1}$. In spite of the different pulse durations and energies, as well as a different value of $\alpha$, all the features visible in Fig. 4 are in good qualitative agreement with those in the simulation of Fig. 3(b). The peak in the visible region represents the axial part of the conical emission which extends to angles up to $8^{\circ}$. The low-intensity supercontinuum in the range between 1 and $1.5 \mu \mathrm{m}$ is more pronounced when the pump energy is increased, in agreement with Fig. 1. The elliptical shape of the spectrum around the fundamental frequency reflects the nature of the nonlinear $\mathrm{O}$ waves generated by spontaneous reshaping of the pump during propagation $[9,12]$.

We can now check that the interpretation of the spectral peak in the visible region shown in Fig. 2 corresponds to the axial part of the conical emission for any pump wavelength. The ratio of the peak velocity for the nonlinear polarization to

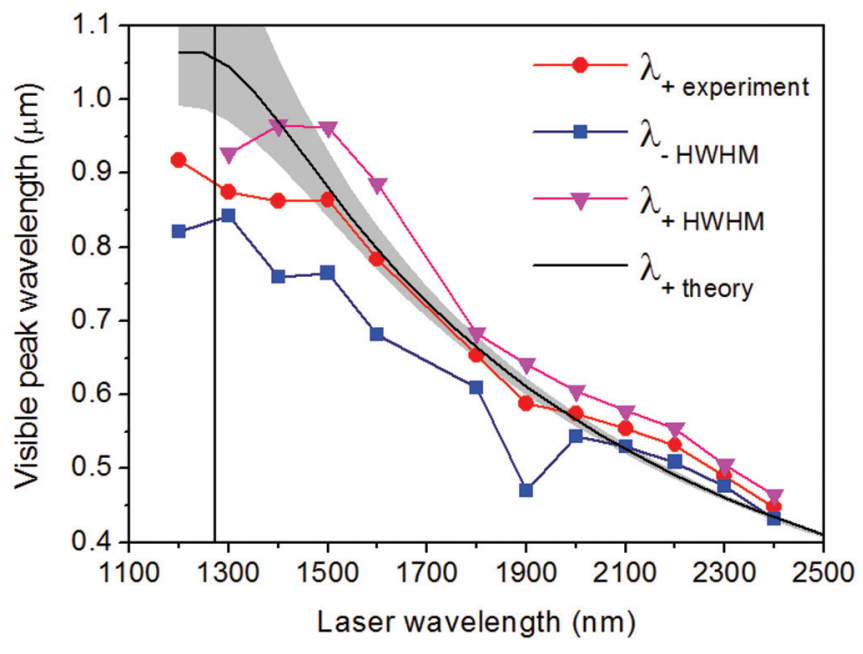

FIG. 5. (Color online) Calculated and measured visible component that is generated during filamentation in a 3-cm-thick fused silica sample as a function of the input pulse central wavelength.

the group velocity was assumed independent of wavelength, i.e., the value for $\alpha$ was assumed to be $1.0002 k_{0}^{\prime}$, with $k_{0}^{\prime}$ varying with the pump wavelength.

The roots of Eq. (2), which represent the intersections of the phase-matched region of the spectrum with the axis $\left(k_{\perp}=\right.$ 0 ), were evaluated by a numerical procedure. For a pump wavelength in the near-IR region, the dispersion landscape is such that three roots can always be found when the nonlinear polarization wave is subluminal $\left(k_{0}^{\prime}<\alpha\right)$. The roots which are closest to the pump belong to an elliptical branch featuring the $\mathrm{O}$ wave, whereas the root at the highest frequency $\omega_{+}$ (wavelength $\lambda_{+}$) belongs to the hyperbolic branch representing conical emission in the visible region.

The spectral position $\lambda_{+}$of the peak in the visible region was compared with the experimental data, as shown in Fig. 5. The black continuous curve corresponds to the wavelength $\lambda_{+}$ of the visible peak as obtained from the model.

From Fig. 2, we measured the central wavelength of the visible peak $\lambda_{\text {+experiment }}$ (red circles), and the full width at half maximum (FWHM) of this peak, delimited by $\lambda_{-\mathrm{HWHM}}$ (blue squares) and $\lambda_{+ \text {HWHM }}$ (purple triangles). The black line was generated with the value $\alpha=1.0002 k_{0}^{\prime}$. The gray area represents new frequencies generated in the visible range, determined by the limits $\alpha=k_{0}^{\prime}$ and $\alpha=1.0004 k_{0}^{\prime}$ in the axial phase-matching conditions. The vertical line represents the zero group velocity dispersion (GVD) wavelength. The departure of the analytical estimation from the measurements near the wavelength of zero GVD is attributed to the narrow range of peak velocities used in Eq. (2). In fact, pulse splitting becomes more prominent at shorter wavelengths, leading to additional polarization waves propagating at different velocities and modifying the position and width of the visible spectral peak. The quantitative agreement between the model and the measurements in the region of anomalous dispersion is very good, thus leading to the conclusion that the phenomena of conical emission accompanying filamentation can be accurately explained by the effective three-wave mixing model, not only in the normal dispersion regime [18,21] but 
also in the anomalous dispersion regime $[11,12]$. In particular, the model accurately predicts the presence of a peak in the visible region that is separated from the supercontinuum in the near infrared. The emergence of this peak cannot be attributed to self-phase modulation alone or to third-harmonic generation.

The formation of the visible spectral peak was observed in Refs. [14-16], and in this article, we interpret its blueshift with increasing pump wavelength. Our experiment differs from that of Ref. [14] in the range of pump wavelengths used as well as the measurement of the conical emission spectrum. The blue spectral peak narrows as the pump wavelength moves further from the zero GVD wavelength. The narrowing also makes the movement of the peak towards shorter wavelengths more easily observable, as reflected in Fig. 2 for pump wavelengths between 2 and $2.4 \mu \mathrm{m}$. Secondly, in Ref. [14] the entire emission cone from the filament was angularly integrated (focused) into the spectrometer. The collection or integration of the entire conical emission may broaden the blue peak and therefore obscure the frequency shift. Differences in the pump pulse energies used between Ref. [14] and our experiment are minor and should not have had a significant influence on the results.

The wavelength and intensity of the visible spectral peak depend strongly on the filament characteristics, and therefore on experimental parameters such as pulse duration, chirp, and focusing geometry. Depending on the dispersion profile of the medium and the initial pulse parameters, the velocity of the pulse intensity peak may vary. This would consequently modify the conical branch of the filament emission and the on-axis emission wavelength in the visible region. A narrow and well-defined visible spectral peak develops if the pulse peak intensity and velocity remain fairly constant over the length of the filament. Conversely, if the velocity of the pulse intensity peak varies significantly during propagation, the visible spectral peak becomes broader as a result of the effective three-wave mixing model applied to the case of a nonuniformly moving material wave. Higher initial peak powers may lead to multiple splitting events and to potentially chaotic multiple collapses [7]. In this case, the "polarization wave" is more complicated but each split pulse can still be interpreted as being associated to a well-defined velocity, leading to a phase-matching relation for each intensity peak. The spectral content in the visible region is then a result of the superposition of the visible peaks generated by each polarization wave. The numerical simulations performed in Ref. [15] indicate that a well-defined visible peak may still emerge in such a condition.

The observation of a spectral peak in the visible region alone does not allow us to infer any conclusion on the occurrence of pulse splitting during filamentation. Our measurements with increasing pump pulse power reported in Fig. 1 show a sharply defined spectral peak, which we interpret as the result of one dominant polarization wave. This scenario is well represented by the numerical simulations reported in Ref. [12]. Pulse splitting is evident in the simulations, but a significantly higher multiphoton absorption compared to Ref. [15] prevented the occurrence of multiple collapses, resulting in an intense subluminal pulse whose intensity remains almost constant over $80 \%$ of the filament length. This results in a visible spectral peak at the same wavelength for the whole range of input pulse powers from 5 to $26 P_{\mathrm{cr}}$. We therefore emphasize that there is a one-to-one correspondence between the central wavelength of the spectral blue peak and the velocity of the material wave. However, there may exist multiple sets of initial pulse parameters, leading to different filamentation dynamics, that can lead to the same final polarization wave with a given velocity.

In this article, we have presented measurements of conical emission for filamentation in the regime of anomalous dispersion. We have identified the mechanisms responsible for the generation of a peak in the visible region which is clearly separated from the supercontinuum around the pump wavelength. We have shown that the effective three-wave mixing model and phase-matching arguments give an accurate explanation for this phenomenon.

The authors acknowledge the funding sources allowing this work: the US Army Research Office MURI Program No. W911NF1110297, the State of Florida, and the ONERA.
[1] A. Couairon and A. Mysyrowicz, Phys. Rep. 441, 47 (2007).

[2] S. L. Chin, S. A. Hosseini, W. Liu, Q. Luo, F. Théberge, N. Aközbek, A. Becker, V. P. Kandidov, O. G. Kosareva, and H. Schroeder, Can. J. Phys. 83, 863 (2005).

[3] C. D’Amico, A. Houard, M. Franco, B. Prade, A. Mysyrowicz, A. Couairon, and V. T. Tikhonchuk, Phys. Rev. Lett. 98, 235002 (2007).

[4] B. Forestier, A. Houard, I. Revel, M. Durand, Y. B. André, B. Prade, A. Jarnac, J. Carbonnel, M. Le Neve, J. C. de Miscault, B. Esmiller, D. Chapuis, and A. Mysyrowicz, AIP Adv. 2, 012151 (2012).

[5] J. Kasparian, M. Rodriguez, G. Méjean, J. Yu, E. Salmon, H. Wille, R. Bourayou, S. Frey, Y. B. André, A. Mysyrowicz, R. Sauerbrey, J. P. Wolf, and L. Wöste, Science 301, 61 (2003).

[6] C. P. Hauri, W. Kornelis, F. W. Helbing, A. Heinrich, A. Couairon, A. Mysyrowicz, J. Biegert, and U. Keller, Appl. Phys. B 79, 673 (2006).
[7] K. D. Moll and A. L. Gaeta, Opt. Lett. 29, 995 (2004).

[8] M. A. Porras, A. Dubietis, E. Kucinskas, F. Bragheri, V. Degiorgio, A. Couairon, D. Faccio, and P. Di Trapani, Opt. Lett. 30, 3398 (2005).

[9] M. A. Porras, A. Parola, and P. Di Trapani, J. Opt. Soc. Am. B 22, 1406 (2005).

[10] A. Saliminia, S. L. Chin, and R. Vallée, Opt. Express 13, 5731 (2005).

[11] D. Faccio, A. Averchi, A. Couairon, A. Dubietis, R. Piskarskas, A. Matijosius, F. Bragheri, M. A. Porras, A. Piskarskas, and P. Di Trapani, Phys. Rev. E 74, 047603 (2006).

[12] M. Durand, A. Jarnac, A. Houard, Y. Liu, S. Grabielle, N. Forget, A. Durecu, A. Couairon, and A. Mysyrowicz, Phys. Rev. Lett. 110, 115003 (2013).

[13] F. Silva, D. R. Austin, A. Thai, M. Baudisch, M. Hemmer, D. Faccio, A. Couairon, and J. Biegert, Nat. Commun. 3, 807 (2012). 
[14] E. O. Smetanina, V. O. Kompanets, S. V. Chekalin, A. E. Dormidonov, and V. P. Kandidov, Opt. Lett. 38, 16 (2013).

[15] E. O. Smetanina, V. O. Kompanets, S. V. Chekalin, and V. P. Kandidov, Quantum Electron. 42, 913 (2012).

[16] E. O. Smetanina, V. O. Kompanets, S. V. Chekalin, and V. P. Kandidov, Quantum Electron. 42, 920 (2012).

[17] M. Kolesik and J. V. Moloney, Opt. Express 16, 2971 (2008).
[18] M. Kolesik, E. M. Wright, and J. V. Moloney, Opt. Express 13, 10729 (2005).

[19] A. Couairon, E. Brambilla, T. Corti, D. Majus, O. de J. RamírezGóngora, and M. Kolesik, Eur. Phys. J.: Spec. Top. 199, 5 (2011).

[20] I. H. Malitson, J. Opt. Soc. Am. 55, 1205 (1965).

[21] D. Faccio, A. Averchi, A. Lotti, M. Kolesik, J. V. Moloney, A. Couairon, and P. Di Trapani, Phys. Rev. A 78, 033825 (2008). 